\title{
Permeable Roads and Pavements for Ground Water Replenishing Water Drinking Concrete
}

\author{
Kongan Aryan, A.K. Gupta, Karnik Agrawal
}

\begin{abstract}
Over-abuse of groundwater has prompted a persistently diminishing groundwater level in the vast majority of the urban areas of India. Growing population, expanding industries, and predicted food demands propose that in upcoming decades, India will suffer from inefficiency to fulfill the requirements of adequate water for its people. In our concrete metropolitans, run-off is a major set back, accounting for reasonable wastage of fresh water. With the aim to tackle the coming catastrophe, this research paper aims at discussing the need and possibility of an efficient and sustainable alternative for managing water wastage. The idea of permeable pavements focuses over the runoff water wastage in our concrete jungles, through pavements, sports court, parking, and other feasible hotspots. Considering the option, it was observed that permeable concrete is an alternative for our impermeable pavements and is effective, efficient, and economical. Hence, with over-exploiting submersibles at action, constant pumping of groundwater, and restricted seepage of water back to its origin, switching to permeable pavement would be a viable opportunity.
\end{abstract}

Keywords: Water Resources; Water Table; Seepage and Run-Off; Permeable Pavements.

\section{INTRODUCTION}

As described by The United Nations, "Water is a social and cultural good, and not merely an economic commodity." Water is the most critical asset and a fundamental ware for the survival of living things. Nourishing arrangements, life stocks, vegetation, producing businesses and social trusts, water is an interlaced and noticeable component of each circle of life.

In the current scenario, while the world predicts the inspiration for the next world war would be water driven, the reality isn't any different. Inefficient supervision, compromising infrastructure, and ignorant ideology has lead to continuous and relentless wastage of the vital resource for our existence. Henceforth, the situation demands a valid concern over securing and preserving the present resource against the upcoming dilemmas, quantitatively and qualitatively.

Our option of permeable pavements is an attempt over the same lines. While our standard asphalt roads and surfaces lead to water runoff and fresh rainwater ending up in flooding sewers, permeable pavements provide an opportunity to combine the dual prospects of sewer and strong pavement to an eco-friendly alternative. Permeable pavements once installed, allows the rainwater to seep down to the strata of

Revised Version Manuscript Received on August 19, 2019.

Kongan Aryan, Department of Civil and Environmental Engineering, Delhi Technical University, Delhi, India (e-mail: akgupta@dce.ac.in).

A.K. Gupta, Department of Civil and Environmental Engineering, Delhi Technical University, Delhi, India (e-mail drkonganaryandcedtu@gmail.com)

Karnik Agrawal, Department of Civil Engineering, Delhi Technical University, Delhi, India. (e-mail: writerkarnik@gmail.com) soil, infiltrating through the pores of the concrete. When opportunity effectively utilized, this can be aid at replenishing our degrading water table in cities, meanwhile, providing an efficient water seepage system at parking lots and possible places.

This research henceforth aims to discuss and consider the need and efficiency of permeable pavements as a feasible alternative on ideal and practical grounds.

\subsection{Upcoming Threat}

Water covers approximately $78 \%$ of our Earth's surface in the form of oceans, seas, icecaps, glaciers, rivers, lakes, groundwater, water vapour etc. However, $97.5 \%$ of the available water on the planet is unfit for human consumption and less probably utilized for other industrial or social activates. Fresh water, rest as called, hence constitutes only $2.5 \%$ of our total water resource available, of which most of it is also trapped and reserved frozen in icecaps. Therefore, only $0.3 \%$ of total water reserve is for use, available in the form of rains, rivers, lakes, ponds, and groundwater.

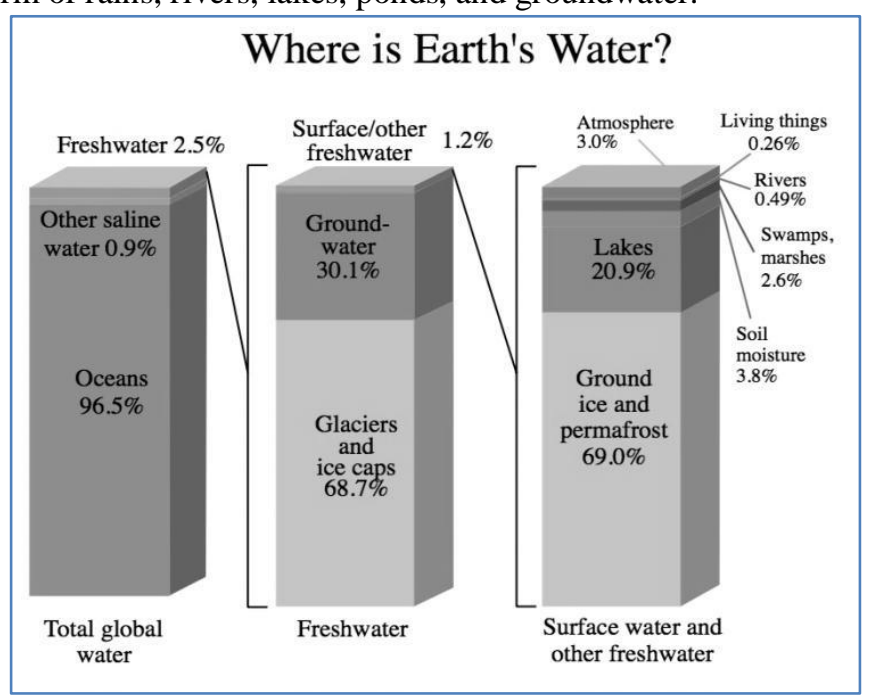

Graph 1. Representation of share of fresh water

Quantitatively, this freshwater resource of $0.3 \%$ is enough to thrive the needs of the existing population of the world. However, uneven distribution of this resource and highly uneven population distribution creates the a real dilemma.

Looking over the facts and data, Asia holding around 60\% of total population controls over only nearly one third of total water resource. Evidently deficient for the present and growing demands of the developing countries, including 


\section{PERMEABLE ROADS AND PAVEMENTS FOR GROUND WATER REPLENISHING WATER DRINKING CONCRETE}

India. In contrary, America is gifted with approximate to half of our fresh water reserve for its one tenth of population share. This situation therefore presents the need of the judicious use of our limited and irrational stake, victimising Asian countries, particularly India, for being vulnerable to water scarcity.

\begin{tabular}{|c|c|c|c|c|c|c|c|}
\hline Réference & Asia & Europe & $\begin{array}{l}\text { Northen \& } \\
\text { Central } \\
\text { Americe }\end{array}$ & South America & Australia & Aficica & Total \\
\hline Population (Y) & 59.63 & 9.89 & 4.8 & 8.53 & 0.56 & 16.59 & 100 \\
\hline Water ( $\left(\mathrm{km}^{\wedge} 3 /\right.$ vear) & $13,508.0$ & $2,900.0$ & $7,770.0$ & $12,030.0$ & $2,4000.0$ & $4,040.0$ & $42,650.0$ \\
\hline Water $\left(y_{0}\right)$ & 31.672 & 6.800 & 18.218 & 28.206 & 5.627 & 9.4725 & 100 \\
\hline
\end{tabular}

Table 1. Distribution of population and fresh water resource (Continent-Wise)

While we don't need to worry about any decrease in the net fresh water available because of the water cycle, our concern still lies with the management of water we hold. India is a gifted country with a range of soil types, vegetation, natural conditions, and topographies. Therefore, water resources also vary in the form of source, state, quality, and quantity throughout the country, major sources being perennial rivers from glaciers, rainfall, and groundwater.

\begin{tabular}{|c|c|}
\hline \multicolumn{2}{|c|}{ Water resources of India (Year 2000) in $\mathrm{km}^{\wedge} 3$} \\
\hline $\begin{array}{c}\text { Estimated annual precipitation (including } \\
\text { snowfall) }\end{array}$ & 4000 \\
\hline Average annual potential in rivers & 1869 \\
\hline Estimated utilisable water & 1123 \\
\hline (i) Surface & 690 \\
\hline (ii) Ground & 433 \\
\hline Water demand $\approx$ utilization & 634 \\
\hline (i) Domestic & 42 \\
\hline (ii) Irrigation & 541 \\
\hline (iii) Industry, energy \& others & 51 \\
\hline
\end{tabular}

Table 2. Total water resources of India (according to year 2000)

Water table or groundwater level is the depth below the surface to the strata of soil with saturation. Groundwater level depends on various natural factors including the nearest abundant source of water like a river or lake, vegetation and terrain in the surrounding area, and environmental conditions. However, in recent decades, artificial factor of over-exploitation of groundwater, and hence degrading groundwater level is a major concern. With use of submersible in the cities and metropolitans of India, people in attempt to sustain near the developing hotspots and urban cities have over exploited the groundwater resource. Overuse of pumps, motors, submersibles, and hand pumps have led to a decrease in water tables around the overpopulated areas, increasing the stress over the limited resource.

Groundwater is replenished every during monsoon season and throughout the year through irrigation channels. However, the even after steps and restrictions imposed by the government, the groundwater depth around the overpopulated hotspots of cities continues to fall farther.

In upcoming future, persistent global warming, water pollution, exploitation, exponentially multiplying population, and in general increasing per capita demand, endures to abuse water resource. Decreasing water table level, in particular, leads to decreased stream flows, dry and arid surroundings, harmed vegetation, and lesser water availability, along with other moral harms. Water demands tends to increase with increase in temperature and decreasing water resources. This leads to overenthusiastic groundwater pumping, creating a vicious cycle of lowering water table and drying contiguous streams.

\subsection{Wastage as Run-Off Water}

Rainfall and many rivers in India are seasonal. This escorts for switching to alternatives like submersible pumps, motors, and hand pumps to fetch water inadequately and incessantly. As population increases and more people migrate to cities for future prospects, these water demands and groundwater extraction tends to increase. Therefore, in present and upcoming future, receding water table is an artificially created continuous phenomenon with deteriorating quantitative and qualitative features of water resource.

Standard asphalt concrete with negligible porosity and network of voids restricts water seeping through the layer. Water hence runs over the top surface in the direction of head loss, mostly and generally ending up in sewers and wastewater disposal systems as they are designed. Sewage inlets around the parking lots, pavements, sports courts, shopping centres, playgrounds, and pool decks are some example. This water flowing over the surfaces instead of seeping into the strata of soils and replenishing saturation and moisture is termed as the run-off water or run-off wastage. While this phenomenon isn't significant around areas with open grounds, in cities with their concrete covers of roads, pavements, and constructions, run-off wastage is the major culprit disconcerting groundwater replenishment.
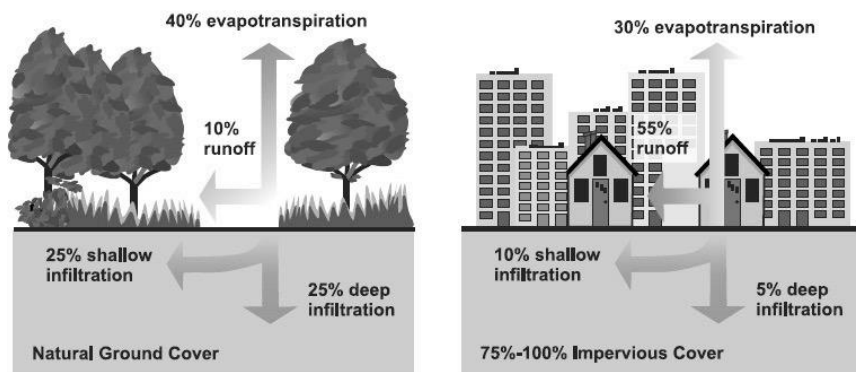

Figure 1. Effect of concrete cover over Run-off and Infiltration

When groundwater is extracted at continuously increasing rate while the levels aren't restored and replenished at the equal rate, the net result of corruption and replenishing turns out to be negative, leading to a gradually receding water table.

Direct and indirect complications with wastage through run-off water constitutes:

1. Fresh potable rainwater being dumped into sewer systems; and capable water source for replenishing groundwater is wasted.

2. Increased sewage system demands with bulk disposal of rainwater and used water, leading to the compromised 
qualitative and quantitative treatment of water. Often sewer water is directly disposed off into streams, barren lands, and wastelands. More sewer leads to more penetration of pollutants into the soil with flow and saturation. This directly leads to more severe effects on the chemical composition of land, land pollution, and harmed vegetation.

\section{METHODOLOGY AND MATERIALS \& RESULTS}

The idea of Permeable Pavements is a modified form of our standard concrete, lacking the finer aggregates leading to the formation of voids allowing water flow. Permeable Pavements, therefore, permit seepage of water along with the characteristic mechanical properties of concrete, including compressive strength. Therefore, instead of run-off wastage, permeable pavements allow permeation of water.

Therefore, this distinctive property of permeable concrete can be utilized for increasing the infiltration and hence replenishment of ground water table.

\subsection{Types of Permeable Materials}

Permeable Materials are available for constructing the permeable pavements and surfaces. Various materials posses of the viable options are:

- Permeable Concrete- Permeable Concrete is the most optimum alternative to standard concrete for producing permeable pavements. In addition to the permeability for infiltration of water, permeable concrete can bear evident traffic and allows higher compressive loads.

- Permeable Asphalt- Permeable asphalt is a sieved form of standard asphalt concrete with specific aggregate sizes used. In general, bigger aggregates provide larger voids and allows more efficient and comfortable flow of water, and hence infiltration.

- Permeable Plastic Lattices- Plastic Lattices are produced according to the demand, fulfilling the threshold requirement for the use. Production of plastic lattices, and hence voids, are therefore $100 \%$ perfect and efficient, promising maximum infiltration lattice is designed for. However, low compressive and load earing strength of plastic grids restrict their use for heavy activities like traffic. Permeable Plastic Lattices are therefore used for domestic conditions, like artificial lawns or restaurant.

- Resin Bound Paving- Resin Bound Paving provides provide strength along with the durability. Aggregates of similar grain size are resin coated for durability and then used for making the mortar for pavement. Similar grain size provides pores and voids enough to allow water infiltration, making resin bound paving a feasible option for light vehicle pass ways and car parks.

\subsection{At Glance: Permeable Concrete}

A United Kingdom based company propagated its product of permeable concrete in 2015. The specifications and features for the concrete being absorption rate of $4 \mathrm{~m}^{3}$ of water per unit time in the first minute, with an average of 600 $\mathrm{dm}^{3}$ per square metre per minute.

Some features of the permeable concrete as boasted by the company, making it a viable option for permeable pavements are: their own characteristic properties, and pros and cons. Some

- At maximum, the infiltration rate can be extended up to $4 \mathrm{~m}^{3} / \mathrm{min} . / \mathrm{m}^{2}$ surface.

- The Load Bearing Capacity and Compressive Strength of about N10 and N20 standards. This provides its incompressible and high load bearing strength features, making it capable for use in construction of car parkings and other light load surfaces.

- With 1.5-3N/mm ${ }^{2}$ Flexural Strength bracket, concrete resists rupture due to shear stress and rough use.

- Porosity and water infiltration can be customized by selecting the grain size distribution. Finer particles are removed partially or completely, producing void spaces ranging from $10 \%$ to $35 \%$ of the volume. Therefore, concrete can be selected and adapted to the requirements, terrains, rainfall, and other essential factors.

\subsection{The System and Set-Up}

The general structure of the permeable pavement system constitutes of three different strata. In most cases, these three beings, the topmost layer of permeable concrete, utilized as the passage for the flow of water and is in active contact with the users; the sub-base aggregate layer constituting gravel, provides the strong and stable foundation to concrete layer and binds the concrete; and the third layer, or impervious layer, used for diverting the flow or storing the infiltrated water. The material, quality, and specifications for the sub-base layer are varied for deciding the infiltration rate of water and manipulating the flow conditions. The impervious layer is used only in certain conditions, otherwise, it is absent, allowing natural infiltration of water to the soil, replenishing the groundwater table and moisture content.

Selection of layers and layout for the permeable pavement system varies with the objective of the system and surrounding conditions. The basic variations and possibilities are:

- $\quad$ Absolute Permeation- This type of system is mostly and solely utilized with an aim of optimal ground water replenishment. In absence of the impervious layer, water infiltrates deeper into the soil without any hassle or restriction. The sub-base aggregate layer is also chosen to allow smooth and efficient passage to water flow.

- Limited Permeation- Type of soil and soil features decide the natural infiltration of water through soil. Therefore, it is sometimes possible that soil restrict flow of water over a threshold velocity of infiltration. In these situations, to avoid overflow or logging, system needs to take into account the time interval required for the flow of infiltrated water into the soil for maximum possible discharge with a factor of safety. This system also lacks the impermeable layer, however, the aggregate sub-base layer is chosen such that it is not capable of draining the water instantly.

- Absolute Restriction- Sometimes, water isn't allowed to infiltrate because of inefficiency of soil to let water go through it, clays, or there are some sewage structures below the surface. In such cases, impervious layer is used to divert the water to some collection tank just after getting infiltrated

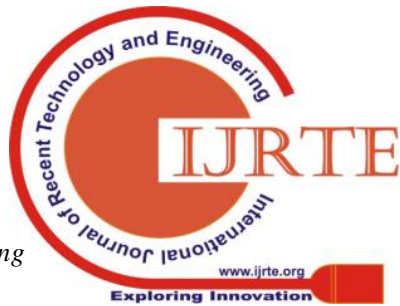




\section{PERMEABLE ROADS AND PAVEMENTS FOR GROUND WATER REPLENISHING WATER DRINKING CONCRETE}

through the sun-base layer of gravel.

As discussed in the case of Absolute Restriction, the permeable pavement system is designed to work like a sub-surface tank which stores the infiltrated water and water evaporates through it with time. In this type of situations, like clay soil or non-draining soils or rock base, sub-base layer demands to be crushed deeper for a thick sub-base layer making water reservoir.

Therefore, pavements are designed properly with respect to the layers, quality of aggregates, and material specifications, considering the surrounding environment, soil type, overload possibilities and factor of safety.

While considering the feasibility of the idea, economic viability is an important factor. In terms of cost of material, permeable pavement systems are slightly expensive than the standard pavements in use. However, on a long term, permeable pavements compensate on that ground too, financially and ethically. Installation of permeable concrete rules out the need for sewage and water disposal systems in the area. Areas like parking lots, car washes, swimming pools, sports court, where chemical drainage isn't an issue, sewer systems and carpet area, both can be replaced by permeable pavement system only. Hence, permeable pavements replace the need for water sewage channels, gutters, outlet channels, and retention ponds, making them more cost efficient than the standard system.

Permeable pavements have the voids and pores which provide passage for the flow of water. With time, these pores are prone to get clogged by the dust particles and grit entering them. This can affect the ease of flow of water for the system as the passage of flow is restricted by the foreign particles. This issue is resolved by the regular maintenance of the permeable pavement system. An industrial vacuum is used to suck off all the blocking dust and grits from the permeable system. Similarly, air blowing or pressure washing is done to remove the blocking of foreign particles. Here, the schedule and duration between successive clean-ups is managed considering the characteristics of the permeable pavement system and the surroundings. Generally, an area with more dust and soil in the air and fewer rain demands cleanings more frequently.

Considering, permeability for the permeable concrete pavement will vary according to the surrounding conditions, characteristics and quality of the material utilized. As quoted earlier, even after taking into account the variations, the average value for allowed infiltration discharge is approximately $600 \mathrm{~mm} /$ minute. According to data varying over a period of 100 years' data, considering the maximum possible rainfall during any storms ever observed around the world, the permeability required is just $5 \mathrm{~mm} /$ minute. This implies that with even more than $90 \%$ pores blocked, the pavement system with work effectively and without any hassle.

Along with all these features, similar to our standard concrete, durability and workability for the permeable pavements are approximately comparable to that of standard impermeable pavements. When properly installed under proper supervision and ensuring the quality, expected life for the pavement is 20 to 40 years, logically decreasing with the rough use.

Therefore, a permeable pavement system offers opportunities for effective sustainable water management and proves to have a viable practical application. Permeable pavement systems can be possibly installed and utilized for sustainable water management and groundwater replenishment, in areas including parking lots, sports courts, pool sides, pavements, car washes, and even driveways.

\subsection{Mechanical Properties and Factors}

\subsubsection{Seepage or Flow Velocity}

For a given dynamic system of soil environment, Darcy's law calculates the flow relations under number of limitations and restrictions. Under uniform and controlled conditions, flow velocity and seepage velocity can be roughly calculated for consideration.

According to Darcy's law, flow velocity for water flowing through a permeable layer of soil under steady conditions is directly proportional to the hydraulic gradient for the flow. The relation of Darcy's velocity with the hydraulic conductivity and gradient is

$$
\begin{aligned}
\text { Darcy's Velocity } & (v) \\
& =\text { Hydraulic Gradient } \\
& \times \text { Hydraulic Conductivity } \\
& v=K \times i
\end{aligned}
$$

In the relation, $K$ defines the hydraulic conductivity or the coefficient of permeability for permeable material. Hydraulic conductivity is defined as flow per unit of driving force and conveys the ease of flow of water through the soil sample. $i$ is called the hydraulic gradient, and is defined as the ratio of pressure head between the two references to the distance along reference points. Also, pressure head is the quantity symbolizing the internal energy of water because of its surrounding.

Flow velocity, or Darcy's velocity is the factual fluid velocity through the soil layer. Flow velocity neglects the surface area occupied by the soil particles and assume as if water flowing through open cross-section. However, seepage velocity is the actual velocity of fluid, the velocity with which water travels through the channels of pores, under experimental approximations.

The factors affecting the velocity of fluid, water in this case, depends on various factors, including the fluidity and density of the fluid, porosity of soil layer, pressure heads and saturation of the soil, and other constants and variable parameters.

Permeability is defined as the representative property of the material or medium to restrict the flow of fluid through pores or the ease offered for flow.

Porosity is the volume of voids to the total volume of the soil. Porosity is the factor associated with the level of packaging for the particles.

The seepage velocity in terms of the flow velocity is related as product of flow velocity, $v$ and the porosity, $\phi$. When porosity is expressed as the ratio of effective cross-sectional area of voids to the total cross-sectional area of sample. 


$$
\text { Seepage Velocity }=\frac{v A}{A^{\prime}}=\frac{v}{\phi}
$$

Hence, for a permeable layer of soil, seepage velocity of flow of water is,

$$
\text { Seepage Velocity }=\frac{K \cdot i}{\phi}
$$

As assumed fluid to be water in this case, the density and fluidity of fluid is for water. For the sake of consideration, porosity for the concrete is taken $25 \%$ as it ranges from $10 \%$ to $35 \%$. Hence, $\phi=0.25$. According to the data from a reliable source, hydraulic conductance varies with the density of the concrete produced.

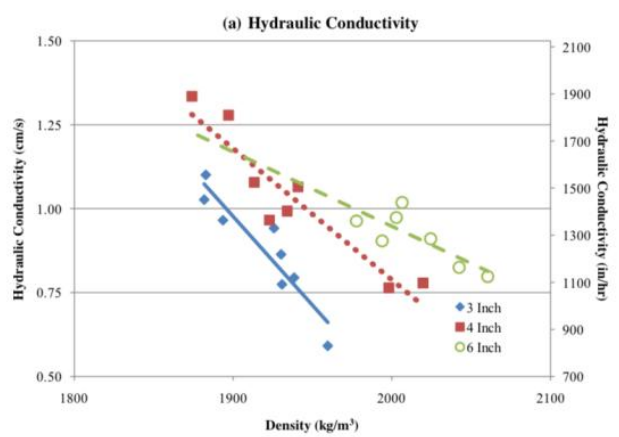

Graph 2. Hydraulic Conductivity Variation

In reference to the graph, considering the probable value of the hydraulic conductance,

$$
K=0.01 \mathrm{~m} / \mathrm{sec}
$$

Therefore, the formula for seepage velocity reduces down to,

$$
\begin{aligned}
\text { Seepage Velocity } & =\frac{K . i}{\phi}=\frac{0.01 \times i}{0.25} \mathrm{~m} / \mathrm{sec} \\
= & (0.4 \times i) \mathrm{m} / \mathrm{sec}
\end{aligned}
$$

Here, $i$ is a variable and depends on factors ranging from groundwater level, moisture content of soil, density, temperature, and hydraulic properties of soil.

\section{RESULT}

Besides the sustainable and eco-friendly action of groundwater replenishment by permeable pavements, other indirect and direct add-ons to the idea are:

- Assists with effective sewage water treatment and lesser load over the functioning of treatment plants. This meanwhile reduces the soil pollution with limited sewage infiltration polluting soil

- Eliminating the sewage water overflow, it contributes to hygiene and safety during the heavy rains and heavy discharge of water.

- Water purifies as it seeps through the different layers of concrete, gravel, and soil, regulating the water quality around the area.

- Comparatively rougher nature of the concrete surface provides a higher coefficient of friction and hence greater grip for vehicles.

- Pores in the roads allow space for rooting of trees ad smaller plants, this assists healthy growth of surrounding plants as well as better binding of concrete to the soil.

- Traces of waters remain in the pores and voids, this water evaporates providing a cooling effect in the surrounding, lowering surface and surrounding temperature.

\section{CONCLUSION}

Finally, considering the idea of permeable pavement systems on the ethical, economical, and efficiency grounds, it can be concluded that 'Water Drinking Concrete' is the viable alternative for our standard pavements.

Our present modern world is suffering from various imminent threats. Whether it be pollution and over-exploitation of resources, global warming, or exponentially growing population, crunched water availability to suffice the increasing demands of our people is a major issue in the field. Managing with our restricted limited water share, sustainable and efficient water management is not an option but a need.

Considering the case of India, and particularly major urban cities, availability of quality water is compromised. Our groundwater resource has been already over-exploited with the aggressive use of submersibles, hand pumps, and motors. Assisting this in the current scenario, our ineffective water and sewer management system let potential freshwater run-off waste into the sewage system. Receding groundwater table seems to be a major menace with the struggle for enough quantity of quality water, and for which we need an immediate effective solution.

In conclusion, facing the viable wastage of water and receding water levels, the idea of permeable pavement systems is a feasible, economical, efficient, and ethical option with unlimited opportunities in hand. Permeable pavement and hence water drinking concrete is the sustainable option to replenish our groundwater levels.

\section{REFERENCES}

1. www.fao.org - Data on growing water demands and consumption

2. https://upload.wikimedia.org/wikipedia/commons/4/46/N atural \%26 impervious_cover_diagrams_EPA.jpg

Pictorial representation for run-off water

3. https://en.wikipedia.org/wiki/Permeable_paving Permeable Paving

4. Brochure by Tarmac Company (Topmix Permeable)

5. http://www.pavementinteractive.org/permeable-pavemen $\underline{\mathrm{ts} /}$

6. Presentation: 'Pervious Concrete- The Concrete that Drinks' by 'PCA Northeast, Cement Shippers Association'

7. 'Cost and Benefit Analysis of Permeable Pavements in Water Sustainability'- ESM Final Paper, by Su-Lin Terhell, Kevin Cai, Dylan Chiu, Johnathon Murphy

8. 'Porous Concrete Pavements: Mechanical and Hydraulic Properties'- McCain \& Dewoolkar https://nptel.ac.in 